\title{
dx.doi.org/10.17488/RMIB.38.1.35
}

\section{Sistema electrónico para reeducación postural estática en tiempo real}

\section{Electronic system for Static Postural Re-education in Real Time}

\author{
A.G. Estévez-Pedraza, R. Martínez-Méndez, J. Rodríguez-Arce, O. Portillo-Rodríguez \\ Facultad de Ingeniería de la Universidad Autónoma del Estado de México
}

\section{RESUMEN}

En este trabajo se presenta un sistema electrónico, basado en la medición de la distribución del peso en los pies, cuyo objetivo es coadyuvar en la evaluación postural. La evaluación postural utilizada por los fisioterapeutas para corregir problemas musculo-esqueléticos derivados por la edad, accidentes, enfermedades, etc. El sistema electrónico construido se validó preliminarmente utilizando videocámaras para corroborar la postura en un sujeto. Con los datos obtenidos se pudo confirmar que el sistema propuesto es capaz de ayudar en la corrección de la postura en tiempo real. La limitación principal de este trabajo es el reducido número de pruebas, por lo que sólo se presentan resultados preliminares. La originalidad de este trabajo reside principalmente en la solución propuesta para evaluar y corregir la postura por medio de un sistema electrónico, novedoso por el método empleado y su portabilidad. Derivado de los resultados presentados se puede concluir que sistema propuesto es capaz de ayudar a corregir la postura en tiempo real.

PALABRAS CLAVE: Reeducación postural, evaluación en tiempo real, sistema electrónico. 


\section{ABSTRACT}

This paper presents an electronic system aimed to assist on the postural evaluation by measuring the weight distribution on the feet. Postural evaluation is commonly used by physiotherapists in order to correct muscle-skeletal problems such as those derived from ageing, accidents, diseases, etc. The developed electronic system was validated preliminary using video cameras to confirm the correct postures of the subject under test. With the obtained data it is possible to corroborate that the proposed system is capable to assist on the correction of the postural position in real time. The main limitation of this work is the reduced number of tests, due to this only preliminary results are presented. The main novelty of this work is the proposed solution to evaluate and help to correct the posture by using a portable electronic device based on force sensors. Derived from the presented results it can be concluded that the proposed system is capable to assist on the evaluation and correction of postural position in real time.

KEYwORDS: Postural reeducation, real-time evaluation, electronic device.

\section{Correspondencia}

DESTINATARIO: Rigoberto Martínez Méndez

INSTITUCIÓN: Facultad de Ingeniería Universidad

Autónoma del Estado de México

DIRECCIÓN: Cerro de Coatepec S/N, Col. Universitaria,

C.P. 50130, Toluca de Lerdo, Estado de México, México

CORREO ELECTRÓNICO: rigo@ieee.org

\section{Fecha de recepción:}

15 de octubre de 2016

Fecha de aceptación:

20 de diciembre de 2016 


\section{INTRODUCCIÓN}

Es común que con el paso de los años y dependiendo del estilo de vida, los músculos pierdan elasticidad, sufran deformidades o se atrofien, Causando con esto desórdenes posturales que derivan en problemas de dolor o malformaciones a corto, mediano y largo plazo. Por otro lado, también existen patologías como la escoliosis, hipercifosis, hiperlordosis, genu varum, genu valgo, genu recurvatum, hallux valgus, acortamiento de extremidades, pie plano, pie varo, fascitis plantar, problemas en la zona lumbar, etc., que también afectan la postura y con ello la calidad de vida de las personas ${ }^{[1]}[2][3]$.

Una de las terapias que más se utiliza, por su éxito en el tratamiento de una mala postura, es la reeducación postural global (RPG). Se trata de una metodología fisioterapéutica basada en la propuesta de Mézières en 1947 y de Philippe Souchard en la década de los 80s y que busca corregir desórdenes musculo-esqueléticos por medio de ejercicios suaves y evaluaciones posturales constantes. La RPG se fundamenta en un análisis minucioso de la anatomía del cuerpo humano ${ }^{[4]}$ observando inclinaciones corporales hacia algún lado, nivel de hombros, escápulas, espinas iliacas, glúteos, rodillas, así como la simetría de la masa muscular y alineación de la columna vertebral y tobillos.

La técnica se basa en los siguientes pasos: impresión general, interrogatorio del paciente, examen local de restricciones y re-equilibrio ${ }^{[5]}{ }^{[6]}$. El tratamiento consiste en posturas de estiramiento activo y global de las cadenas musculares por medio de masajes, movimientos rítmicos activos y ejercicios respiratorios con el fin de recuperar la flexibilidad muscular y la movilidad articular, los cuales favorecen al re-equilibrio de la postura y la desaparición de las tensiones y el dolor ${ }^{[7]}$. El primer paso para definir los ejercicios para la terapia consiste en una evaluación de las alteraciones posturales a nivel musculo-esquelético. Para ello la técnica más precisa es la radiología ya que es capaz de revelar la localización de la zona afectada con exactitud ${ }^{[8]}$. Sin embargo, este método es costoso, además de que expone al paciente y médico a dosis de radiación. Por otro lado, el equipo de radiología no está disponible en cualquier consultorio o clínica debido a su costo.

Para evitar los inconvenientes de ésta técnica (radiación y disponibilidad del equipo) se han desarrollado otros métodos de evaluación que van, desde el simple análisis subjetivo, el cual se basa simplemente en la observación del paciente; el uso de espejos para que el paciente se observe a sí mismo y trate de corregir su postura; básculas en cada pie para medir la distribución de peso (ver la Figura 1); sistemas de video que sustituyen al espejo y que permiten observar los planos sagital y frontal simultáneamente, goniómetros y hasta sofisticados sistemas integrales que incluyen software de análisis postural. Un ejemplo es la spinal analysis machine, la cual consta de una estructura metálica con dos balanzas para los pies, cintas métricas, hilos horizontales y verticales ${ }^{[9]}$, y es usada para detectar desequilibrios posturales por medio de un software. Otro ejemplo es el sistema llamado ADiBAS Posture, el cual utiliza cámaras tipo kinect ${ }^{\circledR}$ con las cuales determina distancias y ángulos corporales, ángulo de Cobb, y al igual que la spinal analysis machine, genera reportes médicos. El costo aproximado de este tipo de sistemas es de 3,000 euros (sin cámaras).

Las desventajas de los sistemas simples, como la observación, los espejos y el video, es que son subjetivos y se requiere de la experiencia y conocimientos del evaluador para hacer un buen análisis. Las básculas proporcionan información cuantitativa pero su utilidad es limitada al evaluar sólo la distribución puntual del peso.

Los sistemas integrales son costosos y requieren de un espacio importante para su colocación y puesta en marcha por lo que suelen usarse sólo en laboratorios o clínicas muy especializadas. 


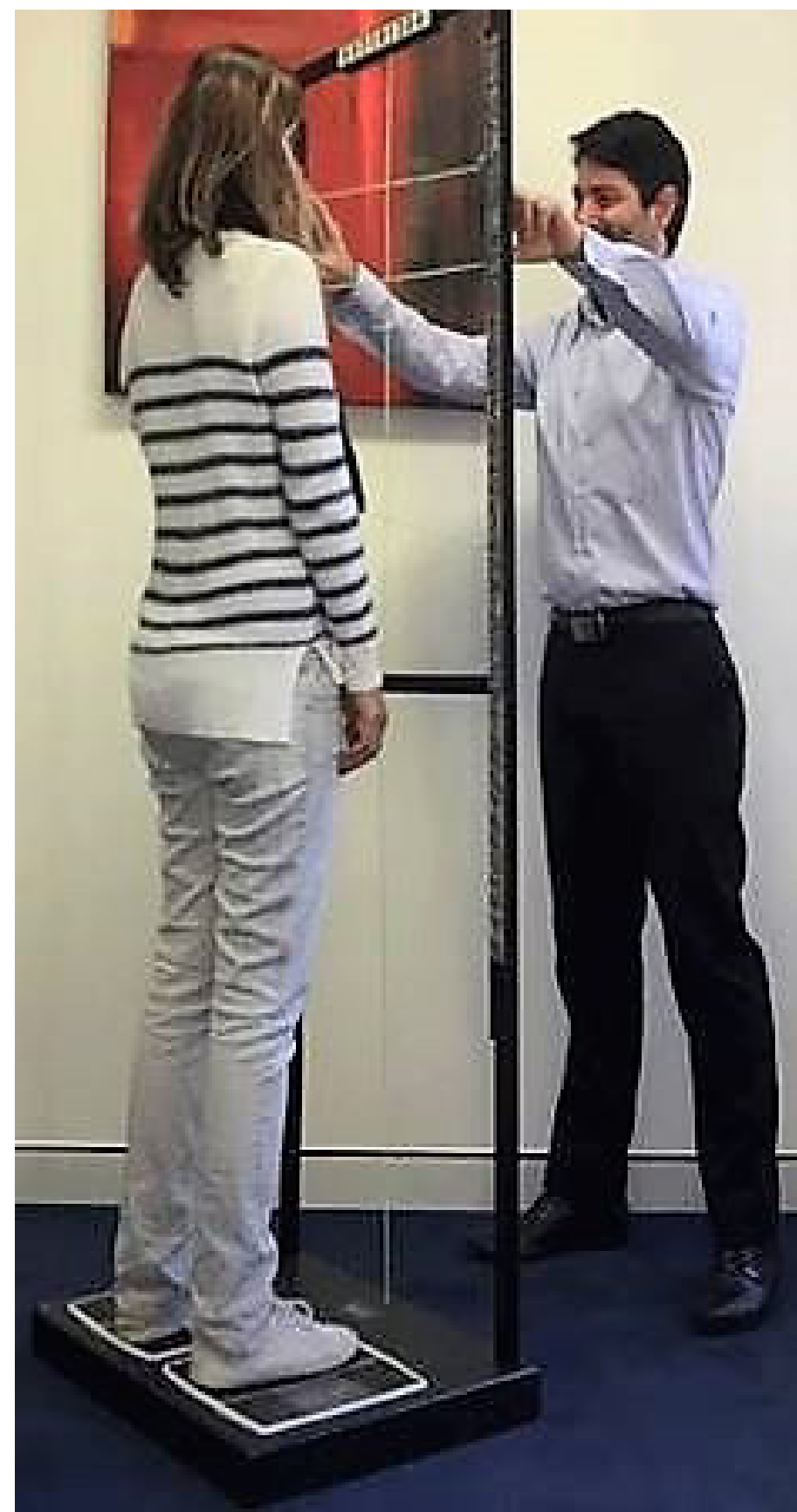

FIGURA 1. Sistema comúnmente usado para la evaluación de la postura ${ }^{[10]}$.

Con el fin de proporcionar una alternativa a la problemática de evaluación y reeducación postural; en este documento se presenta un sistema electrónico portátil para realizar valoraciones posturales basándose en la distribución del peso del cuerpo humano, empleando sensores de fuerza distribuidos en la planta de los pies. El sistema propuesto es capaz de evaluar la postura de pie de un sujeto y proporcionar retroalimentación en tiempo real. Con este sistema se busca ofrecer una alternativa de bajo costo, fácil de usar y que ocupe un espacio mínimo, para que se pueda usar en cualquier consultorio médico o de terapias. Este trabajo se basa en el principio de medición de distribución de peso comúnmente usado en el sistema de básculas, pero con la diferencia de que ahora se mide la distribución de fuerza en cada pie, a diferencia de una báscula común que mide el peso puntual de todo el pie. Se propone y prueba, preliminarmente, la hipótesis de que el análisis de la distribución de peso puede proporcionar información de la postura en posición de pie y estático en tiempo real.

\section{METODOLOGÍA}

\section{A. Construcción del Sistema}

Cuando los fisioterapeutas utilizan básculas como ayuda en la medición de la postura, se basan en la suposición de que un cuerpo bien balanceado o con una postura correcta, debería distribuir el peso de forma simétrica en cada pie. Por lo tanto, una diferencia en el peso medido en cada báscula indica una postura incorrecta y consecuentemente, el paciente debe modificar su postura hasta lograr un peso igual en cada báscula. En este trabajo, a diferencia del sistema de básculas en cada pie, se propone medir la presión plantar en áreas de los pies donde se sabe que se concentra la mayor presión. Esto proporciona información de la distribución no sólo lateral sino también anteroposterior del cuerpo. De acuerdo a Cavanagh [11], el $60 \%$ del peso corporal se concentra en los talones, con presiones no mayores a $140 \mathrm{kPa}$, el $28 \%$ en el antepie y el $8 \%$ en la parte media del pie con presiones no mayores a $60 \mathrm{kPa}$.

Considerando lo anterior se utilizaron sensores de tipo FSR (Force Sensitive Resistor) de la empresa Interlink ${ }^{\circledR}$ los cuales soportan hasta $200 \mathrm{kPa}$, son ligeros, delgados $(0.45 \mathrm{~mm})$ y ampliamente disponibles. 
Se eligieron sensores FSR402 con un diámetro de $18.28 \mathrm{~mm}$ para colocarlos en el área de la primera y cuarta cabeza metatarsiana y sensores FSR406, cuadrados con un área de $39.6 \mathrm{~mm}^{2}$ para colocarlos en el talón (ver la Figura 5). Cabe mencionar que el rango de ambos sensores es el mismo (200 kPa).

Dado que son sensores que presentan un cambio de resistencia inversamente proporcional a la presión aplicada, se conectaron en una configuración de divisor de tensión a las terminales del ADC del Arduino Mega $2560^{\mathrm{TM}}$. Aprovechando la alta impedancia de entrada de los canales ADC se pudieron conectar directamente las salidas de los divisores de tensión. El ADC de la tarjeta Arduino se configuró para funcionar en un rango de 0 a $5 \mathrm{~V}$ usando 10 bits de resolución. Lo anterior proporciona una resolución de $4.8 \mathrm{mV} / \mathrm{bit}$ aproximadamente. En la Figura 2 se observa el diagrama electrónico de conexión de los sensores a la tarjeta Arduino.

El voltaje de salida del divisor de tensión está dado por la Ecuación (Ecuación 1), donde $R x$, en este caso, tiene un valor de $820 \Omega y$ Sx es el valor de resistencia que tendrá cada sensor dependiendo del peso que incida sobre el mismo.

$$
V_{o}=\frac{R x(5 V)}{(R x+S x)}
$$

Con el valor elegido de resistencia y tomando en cuenta el rango de valores de resistencia reportados en la hoja de datos del sensor ( $0.2 k \Omega$ a $100 k \Omega$ ), se obtienen valores de voltaje en el divisor de tensión de $0.04 V$ a $4.02 V$. Además, aun cuando el fabricante de los sensores los recomienda para aplicaciones de 0 a $200 \mathrm{kPa}$, se ha comprobado que realmente pueden medir más allá, aunque, obviamente esto no está garantizado por el fabricante. Sin embargo, con esto en consideración se contempla que el rango completo del ADC sea de 0 a 5 .

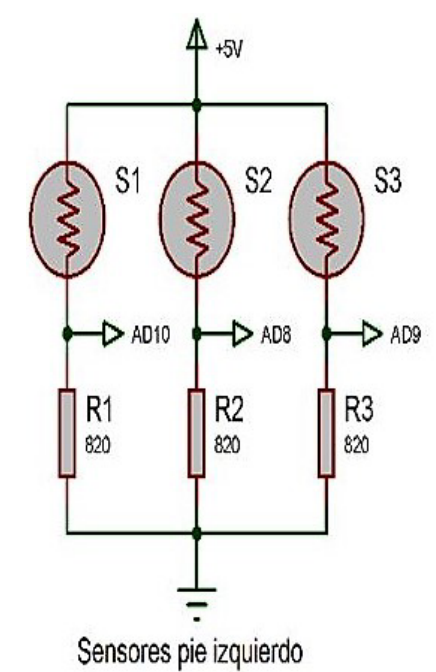

FIGURA 2. Conexión eléctrica de los sensores a la tarjeta arduino.

Es importante mencionar que se eligió la plataforma Arduino por simplicidad en el desarrollo, gracias a sus múltiples librerías para el control de pantallas touch $L C D$, manejo de tarjetas tipo SD. Se utilizó una pantalla genérica LCD TFT a color de 320 x 240 pixeles, con controlador SPFD5408A (Orise Tech) la cual incluye una membrana táctil de tipo resistivo y un zócalo para memoria microSD ${ }^{\mathrm{TM}}$. En la Figura 3 se muestra la pantalla en funcionamiento del sistema propuesto. Se desarrolló un firmware para colocar botones táctiles de inicio y final de la medición. En la pantalla se dibujó la imagen de unos pies y unos círculos rojos que representan la colocación de los sensores y que cambian de diámetro de acuerdo a la presión ejercida en ellos de manera directamente proporcional. El mismo firmware se encarga de almacenar los datos recolectados de los sensores en una memoria microSD ${ }^{\mathrm{TM}}$ a una tasa de muestreo de $5 \mathrm{~Hz}$. Esta tasa de muestreo fue la máxima que se consiguió debido a que la mayoría de los recursos del microcontrolador se ocupan para el manejo de la pantalla LCD, incluyendo temporizadores y módulos de interrupción. La tasa de muestreo es adecuada para la evaluar la postura del pie del sujeto, ya que la tarea de mantener una postura es casi estática y su ancho de banda está por debajo de $2 \mathrm{~Hz}^{[12][13]}$. 


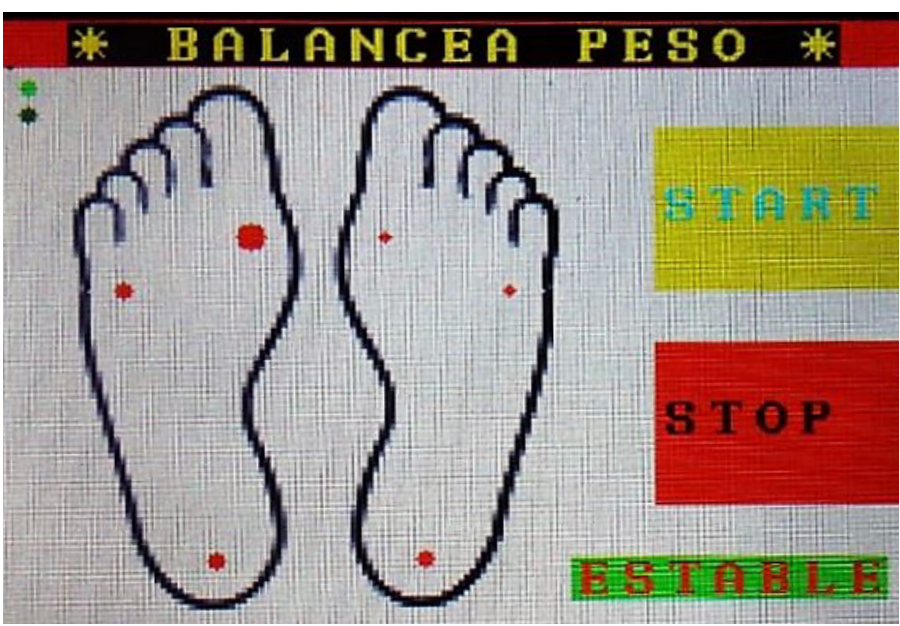

FIGURA 3. Pantalla touch del sistema donde se muestran los botones de inicio y paro de medición, magnitud de la fuerza aplicada a cada sensor y el estado de estabilidad o no.

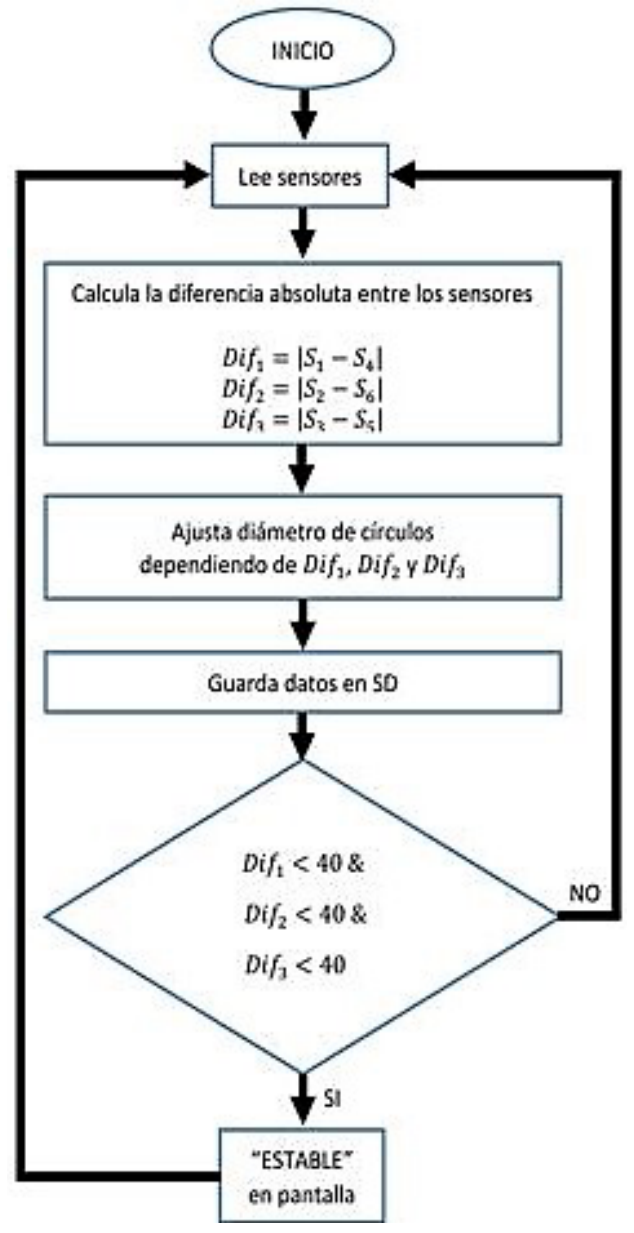

FIGURA 4. Diagrama de flujo simplificado para la determinación de postura correcta. ura y concluir si es estable o inestable se basó en la lectura de cada uno de los sensores y la comparación entre ellos. Es decir, el sensor en el talón izquierdo debía medir lo mismo que el sensor en el talón derecho y la comparación se hace así para cada sensor y su contraparte en el otro pie. Heurísticamente se determinó una diferencia máxima entre el valor de cada sensor de 40 unidades del ADC., como la mejor para determinar estabilidad de postura o no. El diagrama de flujo de la Figura 4 describe en forma simplificada el firmware realizado para el sistema.

\section{RESULTADOS Y DISCUSIÓN}

\section{A. Protocolo de pruebas}

Para realizar las pruebas con el prototipo se colocó un sujeto de prueba, descalzo, frente a una pared con la pantalla del prototipo justo en frente, a la altura de los ojos. Los sensores se colocaron en las zonas de interés de los pies y se fijaron por medio de una cinta adhesiva con el fin de evitar movimientos que pudieran afectar el funcionamiento del sistema (ver la Figura 5).

Se le pidió al sujeto que, utilizando la información mostrada por el dispositivo ajustara él mismo su postura hasta lograr que en la pantalla LCD apareciera la leyenda "ESTABLE", indicando con esto que su postura era correcta.

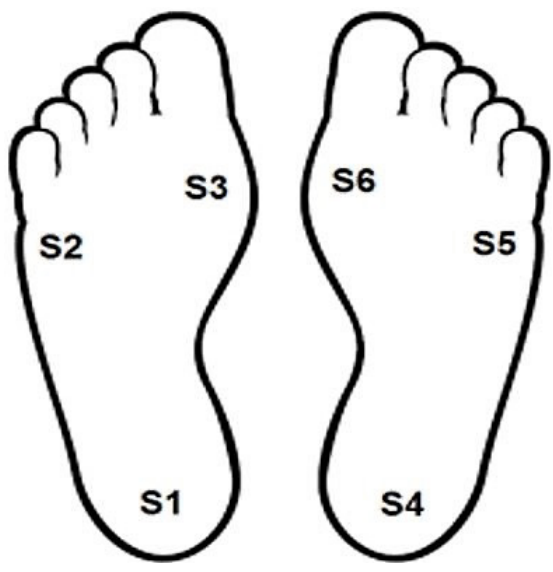

FIGURA 5. Puntos de colocación de sensores en los pies. 
Una vez que el usuario encontró su punto de estabilidad, se le pidió que se balanceara hacia a delante y atrás, a la izquierda y la derecha, con el objetivo de observar la variación de señales en esas posiciones. Para evaluar su postura en todo momento, se colocaron dos cámaras de video, una a espaldas del sujeto y la otra a su costado izquierdo.

La Figura 6 muestra la posición y señales obtenidas cuando el sujeto estaba estable (6A) y después, cuando se mueve hacia atrás (6B), se puede observar como cae la presión en los sensores del antepie (S2, S3, S5 y S6) y aumenta la de los sensores en el talón (S1 y S4). En las imágenes siguientes, la línea verde sobre el sujeto indica una posición correcta y la línea roja indica una desviación.

Para todas las imágenes siguientes las unidades en el eje vertical son una representación en valores decimales del voltaje leído en el sensor (no volts), en el eje horizontal es el número de muestras.

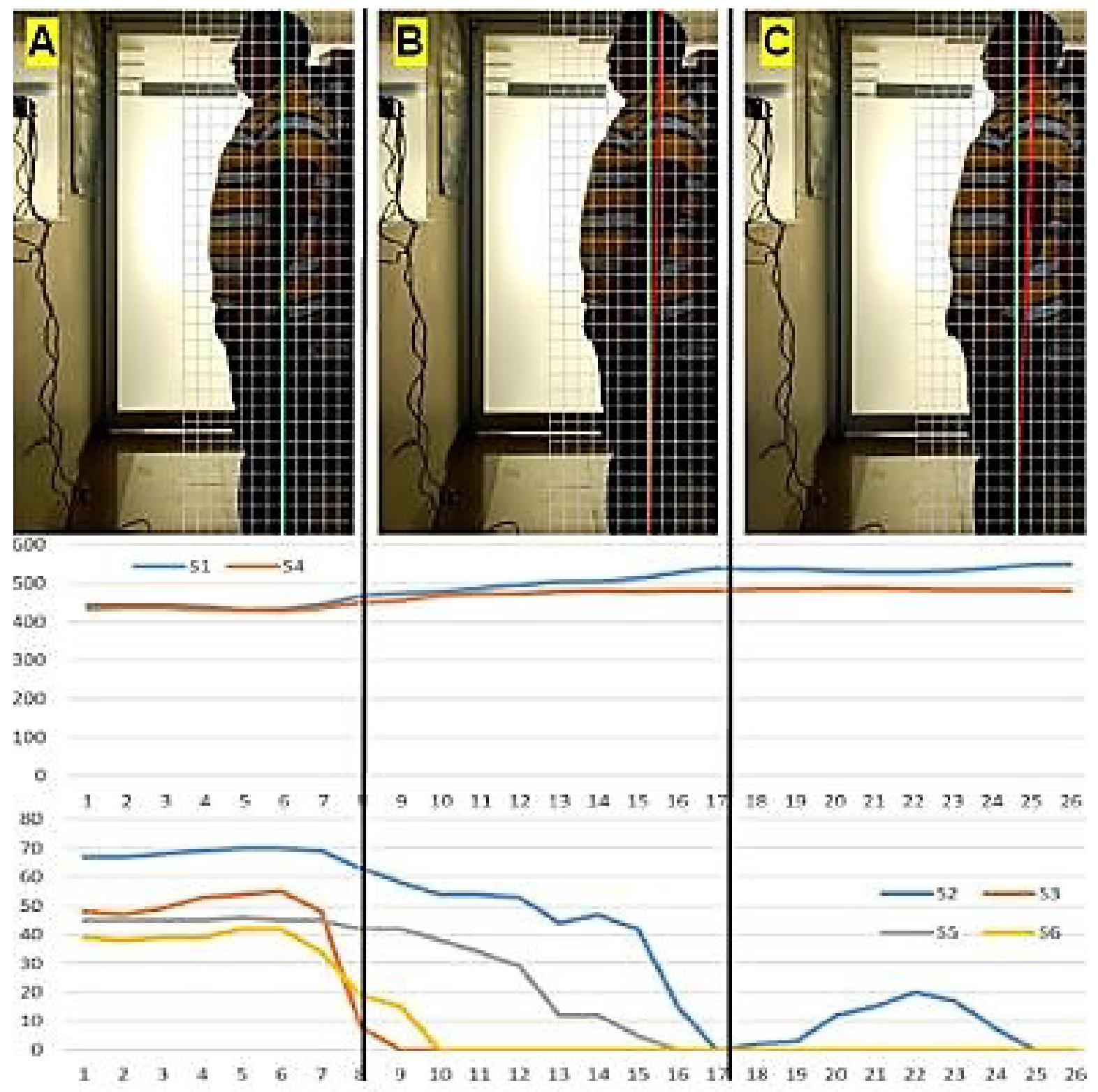

FIGURA 6. A) postura correcta del sujeto. B) Sujeto desviándose hacia atrás, C) Sujeto totalmente desviado en su postura. 
En la Figura 7 se observa el movimiento contrario a la Figura 6. Ahora el sujeto se balancea hacia adelante, causando con ello reducción en la presión en los talones (S1 y S4) y aumento de presión en los sensores del antepie (S2, S3, S5 y S6).
Algo similar ocurre para los movimientos en el plano sagital, hacia la izquierda y derecha. En la Figura 8 y en la Figura 9 se puede observar el comportamiento de las señales en los pies durante estos movimientos respectivamente.

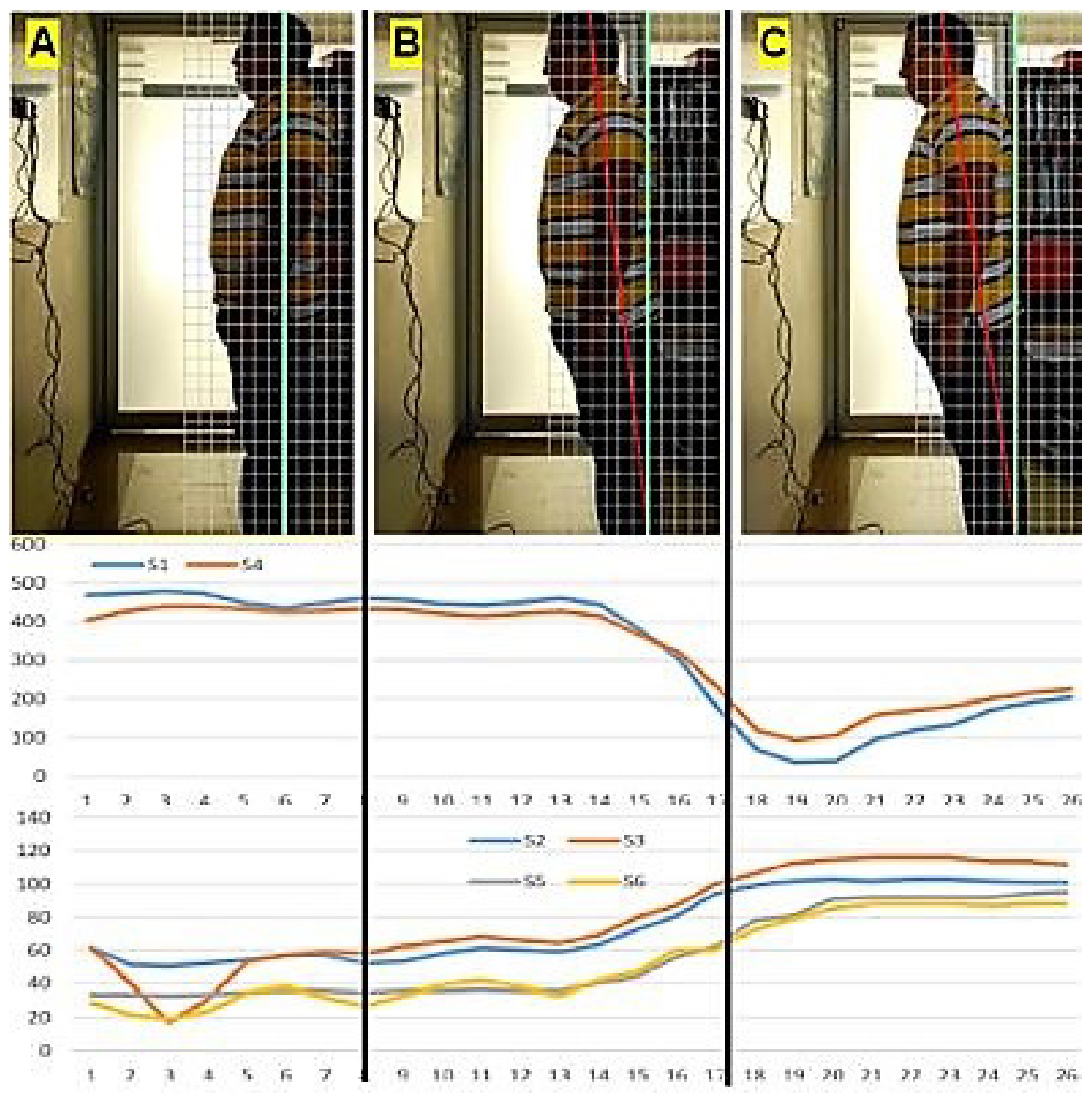

FIGURA 7. A) Sujeto en una postura correcta, B) Moviéndose hacia adelante, c) Postura totalmente desviada hacia adelante. 
Es importante comentar que, siempre que el sujeto lograba colocarse de manera que el dispositivo indicaba una posición "ESTABLE", esto se podía corroborar con imágenes. De acuerdo con la guía de evaluación postural ${ }^{[14]}$, una correcta posición se considera cuando se puede trazar una línea recta que pase por delante del maléolo externo, delante de la articulación de la rodilla y cadera, por la articulación del hombro, por el meato auditivo externo y detrás del vértice de la sutura coronal, lo anterior visto en el plano lateral. Para la vista frontal, se traza una línea recta imaginaria que divide al cuerpo en dos y se trazan líneas horizontales que deben pasar por las clavículas, las crestas iliacas, la parte superior del fémur y las rótulas.

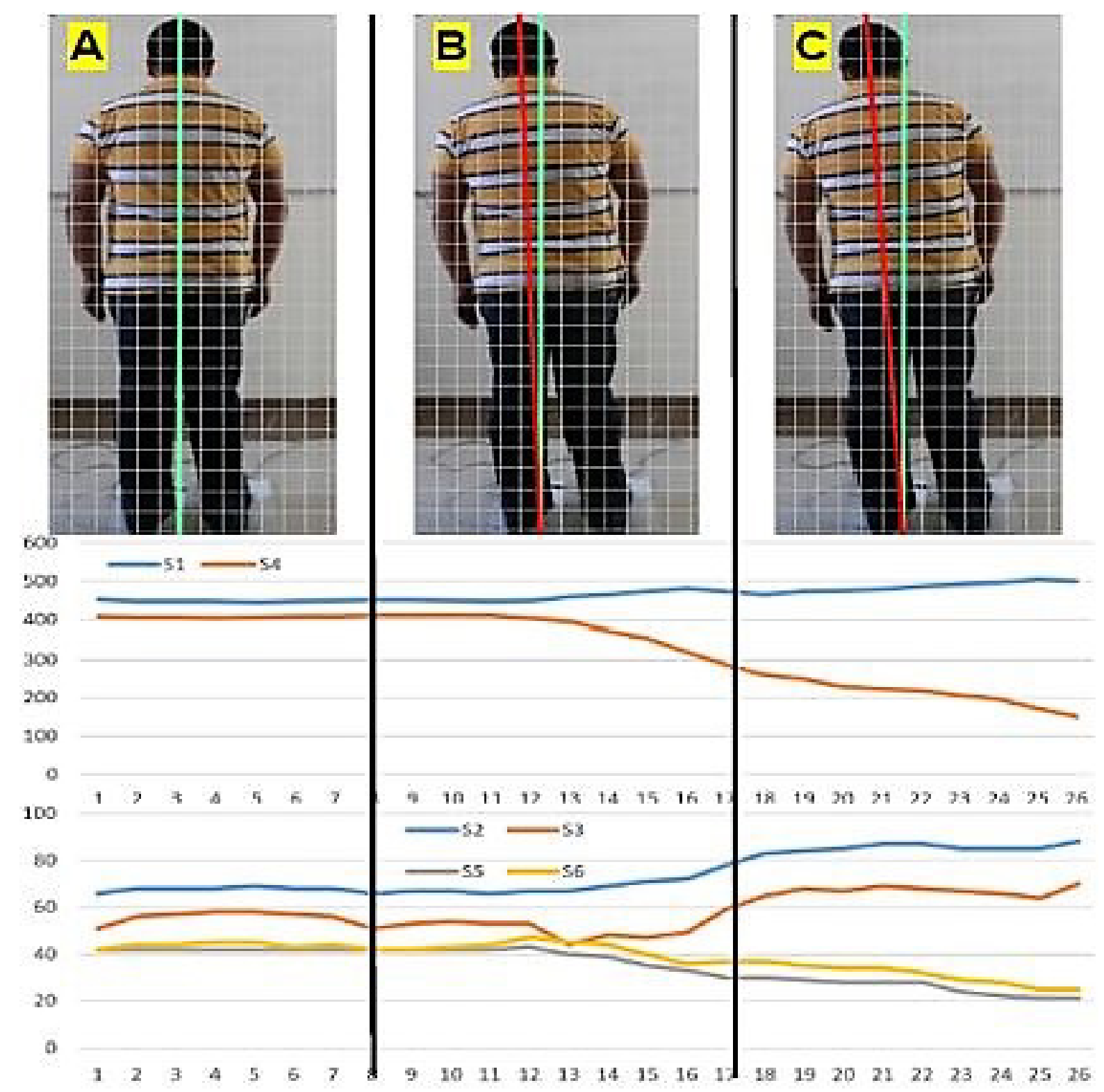

FIGURA 8. A) Postura estable, B) Moviéndose, C) Inestable inclinado hacia la izquierda. 


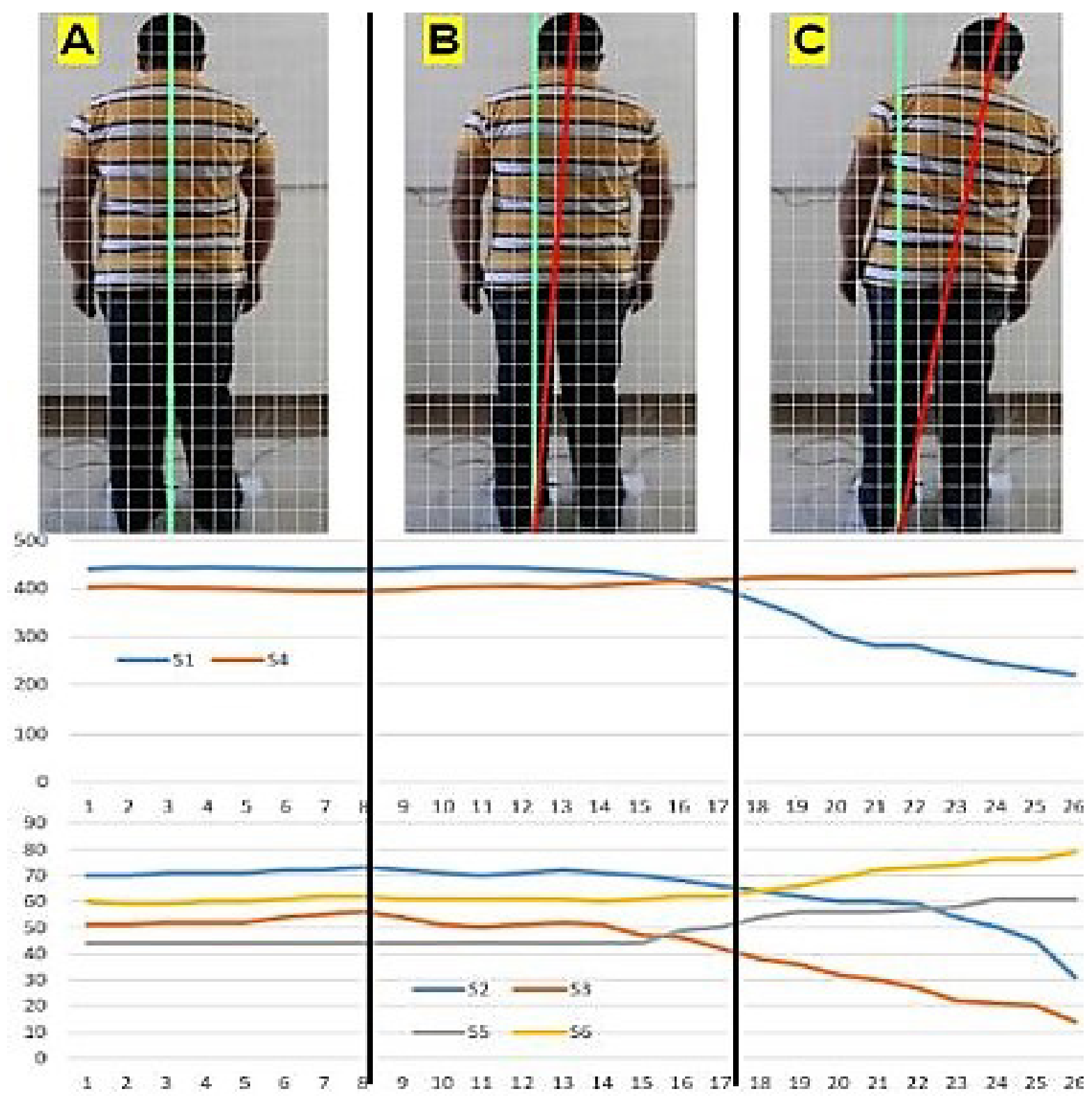

FIGURA 9. A) Postura estable, B) Moviéndose a la derecha, C) Inclinado totalmente a la derecha.

\section{B. Discusión}

De acuerdo a lo observado en las imágenes y comparando con las señales obtenidas de la posición, se observa una clara correspondencia. Esto era de esperarse debido a la colocación de sensores y a los movimientos realizados. Lo más importante es que, por medio de la medición de estos puntos y con el algoritmo presentado, es posible determinar un punto "ESTABLE" que, de acuerdo con las imágenes tomadas de los videos concuerda con una postura correcta. En las figuras y con ayuda de las líneas grises es posible ver que las imágenes del sujeto estable coinciden aproximadamente con estas restricciones para una postura correcta. Si bien, es cierto que para validar completamente a este dispositivo se deben realizar más pruebas, además de comparar los resultados con los de un método tradicional ayudados por expertos en 
el área, portando la vestimenta adecuada para observar los puntos de interés. Esa será la segunda etapa de este proyecto. Con los datos obtenidos en dichas prue- bas futuras se pretende formalizar el algoritmo para garantizar que el sistema funcione independientemente del tipo de complexión del sujeto.

\section{CONCLUSIONES}

En este trabajo se ha presentado un dispositivo capaz de determinar el momento en que se ha logrado una postura correcta. Se presentan resultados experimentales que validan su funcionamiento. Una de las principales ventajas del dispositivo es su capacidad de proveer información en tiempo real de la postura. Esta herramienta podría ser útil, no solo para evaluar a los pacientes en clínicas o consultorios sin la necesidad de más equipos, sino también, puede ser útil para que el mismo paciente compruebe su postura en su mismo hogar, antes y después de realizar la terapia recomendada por los especialistas, con el fin de hacer un seguimiento más preciso de su avance. El dispositivo presentado es portátil, ligero y fácil de usar. 


\section{REFERENCIAS}

[1] L. Gaona, "Valoración de las alteraciones posturales", 2012. [En línea]. Disponible en: http://www.efisioterapia.net/articulos/ valoracion-de-las-alteraciones-posturales [Consultado: 02-jul2016].

[2] A, García-González et al., "Eficacia del método Reeducación Postural Global (RPG) para el tratamiento de la fascitis plantar en adultos entre 40 y 50 años", Univ. Pontif. Madr., pp. 53-59, mar. 2012. ISBN : 978-84-939918-0-7.

[3] F. Bonetti et al., "Effectiveness of a 'Global Postural Reeducation' program for persistent Low Back Pain: a non-randomized controlled trial”, BMC Musculoskelet. Disord., vol. 11, pp. 285, dic. 2010. DOI : 10.1186/1471-2474-11-285

[4] “El Método RPG | Asociación Española RPG”. [En línea]. Disponible en: http://www.rpg.org.es/. [Consultado: 05-jul-2016].

[5] P. E. Souchard, RPG. "Principios de la reeducación postural global". Editorial Paidotribo, 2005. ISBN : 9788480198301 . DOI : 10.1016/ $\underline{\text { S0211-5638(06)74022-6 }}$

[6] "Definición y Principios"| Asociación Española RPG". [En línea]. Disponible en: http://www.rpg.org.es/. [Consultado: 05-jul-2016].

[7] “Método Mézières"| Neus Duran Serrano. [En línea]. Disponible en: http://neusduran.com/metodo-mezieres. [Consultado: 05-jul2016].

[8] "Software de diagnóstico y medición postural 3D", physicaltech. com. [En línea]. Disponible en: http://www.physicaltech.com/adibas-posture/. [Consultado: 06-jul-2016].
[9] Á. Olaru, J. Parra Farré, y R. Balius, "Estudio de validación de un instrumento de evaluación postural (SAM, spinal analysis machine)", Apunts Med. LEsport Castell., vol. 41, núm. 150, pp. 5159, abr. 2006. DOI : 10.1016/S1886-6581(06)70010-X

[10] Sydney Wellness Chiropractic, "Postural test with Spinal Analysis Machine", Sydney Wellness Chiropractic. [En línea]. Disponible en: http://www.sydneywellnesschiropractic.com.au/p roduct/new-patient-first-consultation/.[Consultado: 18-ago-2016].

[11] P. R. Cavanagh, M. M. Rodgers, y A. Iiboshi, "Pressure distribution under symptom-free feet during barefoot standing”, Foot Ankle, vol. 7, núm. 5, pp. 262-276, abr. 1987.DOI: $\underline{10.1177 / 107110078700700502}$

[12] R.-J. Cherng, H.-Y. Lee, y F.-C. Su, "Frequency spectral characteristics of standing balance in children and young adults", Med. Eng. Phys., vol. 25, núm. 6, pp. 509-515, jul. 2003. DOI: 10.1016/S13504533(03)00049-3

[13] H. G. Williams, B. A. McClenaghan, y J. Dickerson, "Spectral characteristics of postural control in elderly individuals", Arch. Phys. Med. Rehabil., vol. 78, núm. 7, pp. 737-744, jul. 1997. DOl: 10.1016/50003-9993(97)90082-4

[14] Roberto Navarrete Aedo, “Guía de evaluación postural”. Escuela de salud DuocUC. [En línea]. Disponible en: http://biblioteca.duoc.cl/ bdigital/Documentos_Di gitales/600/610/41122.pdf [Consultado: 04-jul-2016]. 